\title{
Outcome of living donor kidney transplantation: a single center experience from South India
}

\author{
Vishrut Khullar ${ }^{1}$, Pradeep Shenoy $\mathrm{M}^{2}$ \\ ${ }^{1}$ Department of Internal Medicine, K.S. Hegde Medical Academy, Mangalore, India \\ ${ }^{2}$ Department of Nephrology, K.S. Hegde Medical Academy, Mangalore, India
}

Background: Kidney transplantation remains the treatment of choice for patients with end-stage renal disease (ESRD). However, challenges faced by nephrologists in post-kidney transplant recipients in developing countries are more, mainly due to increased risk of infections which can affect the outcome of such patients. Our objective was to determine the outcome of living donor kidney transplantation including post-operative complications and survival of transplant recipients.

Methods: We conducted a retrospective study of living donor kidney transplantation done in our center Justice K.S. Hegde Charitable Hospital in Mangalore which is a tier two city in India and kidney transplantation was recently started at our center. The transplants done between January 2011 and December 2019 were included. The patient details including out-patient and in-patient records were used to determine recipient and donor characteristics and outcome variables.

Results: Of 34 transplants carried out, 24 (70.6\%) were males and 10 (29.4\%) were female recipients. The mean age was $34 \pm 11.37$ years. Mean duration of hemodialysis was 17.4 months. Cause of ESRD in recipients was unknown in 20 patients $(58.8 \%)$ followed by immunoglobulin A (IgA) nephropathy in three patients (8.82\%). Majority of donors were females (85.3\%). Most common complication seen was urinary tract infections in $32.4 \%$ of the recipients. Acute rejection was seen in four cases $(11.8 \%)$ which were proved by biopsy. Six patients $(17.6 \%)$ had expired while the remaining $28(82.4 \%)$ were alive at time of analysis. The most common cause of death was infections in five (83.3\%) out of six deaths. Of the 28 cases who survived, $23(82.1 \%)$ had functioning grafts and remaining five had been restarted on hemodialysis. The 3-year chance of survival of recipients was $91.17 \%$ and 5 -year chance of survival was $82.35 \%$.

Conclusions: Living donor kidney transplantation is a good option for patients with ESRD even though it is associated with complications, most commonly infections.

Corresponding author: Vishrut Khullar

E-mail: vishrut.khullar@gmail.com

(C) The Korean Society for Transplantation

This is an Open Access article distributed under the terms of the Creative Commons Attribution Non-Commercial License (http://creativecommons.org/licenses/by-nc/4.0/) which permits unrestricted non-commercial use, distribution, and reproduction in any medium, provided the original work is properly cited. 